\title{
A case of verbal and emotional prosody processing dissociation after a right temporal venous infarct
}

\author{
Julie Bourgeois-Vionnet ${ }^{1}$, Annie Moulin ${ }^{2,3}$, Marc Hermier ${ }^{4}$, Agathe Pralus ${ }^{2,3}$, \\ Barbara Tillmann ${ }^{2,3}$, Anne Caclin ${ }^{2,3}$, Norbert Nighoghossian ${ }^{1}$
}

\section{Corresponding author: ${ }^{*}$ Norbert Nighoghossian} norbert.nighoghossian@chu-lyon.fr

1 Stroke Department, Hôpital Neurologique Pierre Wertheimer, 69677 Bron, France

2 Lyon Neuroscience Research Center; CNRS, UMR5292; INSERM, U1028, F-69000 Lyon, France

3 University, Lyon 1, France

4 Neuroradiology Department, Hôpital Neurologique, Hospices Civils de Lyon, Bron, France

This version of the manuscript has been peer-reviewed and accepted for publication in Neurological Sciences.

The publisher's version is available online, since the $4^{\text {th }}$ of January 2020 , at:

https://doi.org/10.1007/s10072-019-04175-w and https://doi.org/10.1007/s10072-020$\underline{04259-y}$

The original article was published online with missing authors :

https://doi.org/10.1007/s10072-019-04175-w.

A correction has been issued, with added authors: Barbara Tillmann and Anne Caclin: https://doi.org/10.1007/s10072-020-04259-y

In the original article, Anne Caclin and Norbert Nighoghossian contributed equally to this manuscript.

\section{Reference:}

Bourgeois-Vionnet, J., Moulin, A., Hermier, M., Pralus, A., Tillmann, B., Caclin A., Nighoghossian N. A case of verbal and emotional prosody processing dissociation after a right temporal venous infarct. Neurol Sci 41, 737 (2020). doi:10.1007/s10072-020-04259-y 


\title{
A case of verbal and emotional prosody processing dissociation after a right temporal venous infarct
}

\author{
Bourgeois-Vionnet, J., Moulin, A., Hermier, M., Pralus, A., Tillmann, B., \\ Caclin A., Nighoghossian N.
}

Letter to the editor

\section{Dear Editor:}

The human voice contains, within its acoustic structure, a wealth of information on the speaker's emotional state that are perceived with remarkable accuracy, in addition to verbal information per se [1]. Specific cortical areas within the upper bank of the superior temporal sulcus (STS) are involved in voice perception [2]. Although voice-selective regions were reported in the STS of both hemispheres, there seems to be a right-hemispheric asymmetry [3]. Abnormal voice perception after stroke is likely underestimated as comprehensive neuropsychological assessment is rarely performed; here, we report a case of emotion perception disorder for voices after a venous infarct involving these specific STS areas, associated with speech-in-noise and musical short-term memory deficits.

A 33-year-old right-handed woman without prior medical history was referred to our stroke department due to severe headache for 10 days. On admission, neurological examination revealed a left visual extinction. Brain MRI showed a right lateral sinus thrombosis associated with a right temporal hemorrhagic venous infarct involving the superior, middle, and inferior temporal gyri (Fig. 1). Etiological workup was negative. She received anticoagulation treatment and headaches vanished within a week.

During her hospital stay, the salient feature of her complaints consisted in a very unusual perception of voices. Conversations were perceived as tragic and, overwhelmed with emotion, she frequently broke into tears. She had the feeling that nurses and medical team members were permanently crying when talking to her. Voices on television were similarly distorted. In contrast, word understanding as well as writing and reading capacities were not affected. In light of these symptoms, we performed neuropsychological tests in two sessions, 5 and 17 days after hospital admission assessing various facets of auditory perception and cognition: emotion, speech perception in different kinds of noise background, and auditory short-term memory of both verbal and musical materials. Assessment was performed after patient informed consent delivery according to neuroscience ethical committee rules and following the agreement of committee for the protection of person's CPPP.

To study emotional prosody perception, sentences of neutral semantic content, pronounced by actors expressing various emotional states, were presented. The patient was asked to recognize the emotion by choosing one of five categories (i.e., joy, sadness, fear, anger, and neutral) and to rate the intensity of the selected emotion on a scale from 1 to 5 (except for "neutral"). The patient's recognition scores (Fig. 2a) were in general lower than those of control participants ( $N=18$, age (mean $\pm S D)=35 \pm 15)$. Correct emotion categorization was only $80 \%$ for the patient, compared with $95 \%$ on average in control participants (mean of the control group-2SD $=80.4 \%$ ). Joy 
recognition was particularly low ( $50 \%$ vs. $98 \%$ on average in controls). Emotion intensity ratings were in the normal range except for a slight reduction for fear stimuli.

This emotion perception test in sentences was repeated 2 weeks later and completed with emotion perception tests in short vowels, music, and faces in the second testing session. Emotion categorization performance was improved and reached normal performance also for joy for sentences, but some deficit was observed for joy in the vowel material (Fig. 2b, c). In addition, emotion categorization was normal for faces and music, as well as intensity ratings for the four types of stimuli tested (i.e., sentences, vowels, faces, and music). Emotion intensity ratings were in the normal range except for a slight reduction for fear stimuli.

Word perception was tested using a 4-alternative forced-choice paradigm, where the patient had to choose, among 4 pictures, which picture matched the word presented at a comfortable loudness level in one ear. The test was repeated for each ear in three different noise environments: silence, cocktail party noise (mix of voices), and speech noise (random noise of the same frequency spectrum as the cocktail party noise) with a signal-to-noise ratio of $-3 \mathrm{~dB}$. Results on day 5 (Fig. $2 \mathrm{~b}$, data from $n=20$ controls, age (mean $\pm S D)=24 \pm 3$, are presented for comparison) showed normal speech perception for both ears in silence (scores greater than 98\%) and speech-noise conditions (scores above 87\%). However, an anomalous asymmetry was obtained specifically in cocktail party noise, with only $45 \%$ correct responses for the left ear versus more than $85 \%$ for the right ear. This was accompanied by a clear pathological score on a short version of the Speech, Spatial, and Qualities of Hearing (SSQ) scale that evaluates hearing deficits in everyday situations on a scale from 0 to 10 . Specifically, the patient scored 4.2 (below the cut-off score of 8.4) on the speech subscale that evaluates speech perception in everyday situations, and 9.8 (normal score) on the qualities of hearing subscale that evaluates naturalness of perceived non-speech sounds. Two weeks later (day 17 ), this auditory deficit almost fully recovered, reaching $85 \%$ of correct answers for the left ear on the cocktail party speech perception test (Fig. 2b), and a normal SSQ speech score of 8.6 .

Finally, to assess auditory short-term memory on the second testing session (day 17, Fig. 2c, d) trials with two sequences of auditory stimuli composed of four items each were presented to the patient, with $1 \mathrm{~s}$ of silence between the two sequences. The patient was asked to judge whether the two 4 item sequences were identical or different. This test was performed with both verbal material (spoken syllables) and musical tones, allowing for the assessment of verbal and nonverbal auditory short-term memory performance with exactly the same task structure. The patient's performance was normal for verbal short-term memory $\left(d^{\prime}=2.42\right.$, mean $d^{\prime}$ in control participants $(n=15$, see $(10)=2.37)$, but a slight deficit was observed for musical short-term memory $\left(d^{\prime}=2.38\right.$, mean $\mathrm{d}^{\prime}$ in control participants $=2.92$, mean $-2 \mathrm{SD}=2.40$ ).

Speech perception and musical short-term memory impairment have resolved within 1 month.

This case shows an impressively specific auditory deficit pattern: (1) a deficit in emotional prosody recognition in sentences in the acute stage, in particular for joyful stimuli; (2) a deficit in speech perception present only with noisy background of a mix of voices; and (3) a deficit in musical shortterm memory.

In contrast, speech understanding and verbal auditory short-term memory were normal. Emotion categorization was also normal for faces and music, ruling out the possibility of global anhedonia. To sum up, in the auditory domain, verbal processing (phonological and semantic content; verbal short-term memory) is preserved whereas the processing of information unrelated to language 
information per se was impaired: emotional prosody, speech-in-speech perception, and musical short-term memory.

The association of prosody perception deficits and speech-in-speech deficits suggests a common mechanism underpinning speech perception in cocktail party situation and prosody perception. The finding of normal musical emotion categorization should be interpreted with caution, as the musical stimuli used were of rather long duration $(20 \mathrm{~s})$, more comparable with sentences (mean duration of $1.5 \mathrm{~s}$ ) for which emotion categorization had recovered at day 17 , than vowels (400 ms).

In this patient, the ischemic injury may have affected a network within the temporal cortex that processes both pitch and prosody. The processing of these information requires a bilateral frontotemporal network [4], with relative specialization of the right hemisphere for emotional prosody perception. The reconstruction of the patient's lesion (Fig.3) revealed that the lesion encompassed voice-selective regions along the superior temporal sulcus where a specific activation, for vocal sounds than for non-vocal sounds, was recorded [5]. However, this interpretation could be labeled as speculation and additional functional imaging data of selective temporal voice area involvement might provide more insight into the pathophysiological mechanism.

Conflict of interest : The authors declare that they have no conflict of interest.

Ethical approval :This work was approved by ethical committee for research involving human participants.

Informed consent : Informed consent was obtained from the patient.

\section{References}

1. Doehring DG, Bartholomeus BN (1971) Laterality effect in voice recognition. Neuropsychologia. 9(4):425-430

2. Belin P, Zatorre RJ, Lafaille $\mathrm{P}$, Ahad P, Pike B (2000) Voice-selective areas in human auditory cortex. Nature. 403(6767):309-312

3. Witteman J, van ljzendoorn MH, van de Velde D, van Heuven V, Schiller NO (2011) The nature of hemispheric specialization for linguistic and emotional prosodic perception: a metaanalysis of the lesion literature. Neuropsychologia. 49(13):3722-3738

4. Frühholz S, CeravoloL, Grandjean D (2012)Specificbrain networks during explicit and implicit decoding of emotional prosody. Cereb Cortex N Y N 1991 22(5):1107-1117

5. Belin P, Bodin C, Aglieri V (2018) A « voice patch » system in the primate brain for processing vocal information? Hear Res 366:65-74 


\section{Figures}
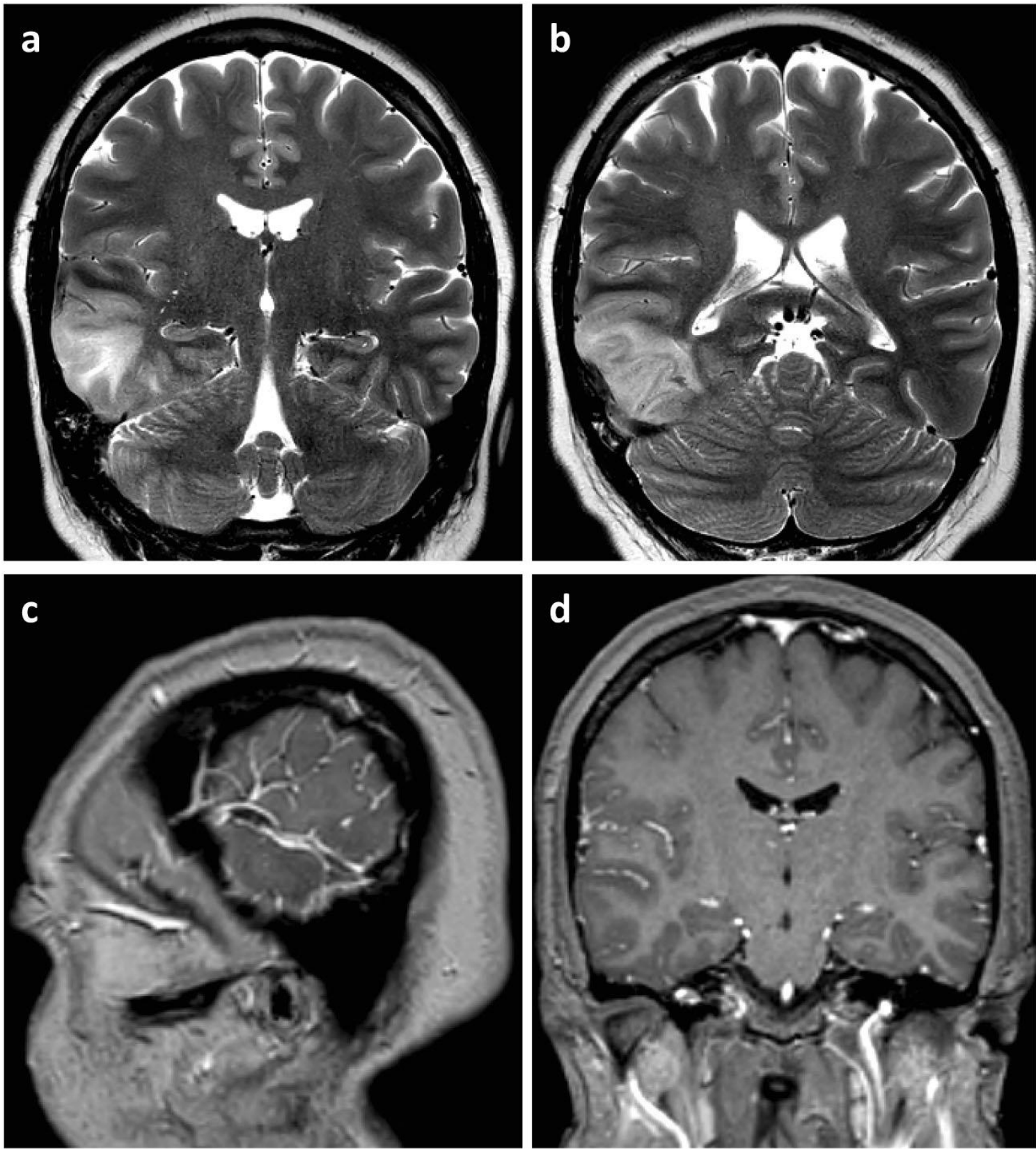

Fig. 1 a, b Coronal T2 slices showing an hyperintense corticalsubcortical lesion involving the inferior part of the right superior temporal gyrus and the middle and inferior temoral gyri, consistent with a venous infarct. c, d Sagittal and coronal postcontrast T1-weighted views depicting a thrombosis of the inferior anastomotic vein of Labbé. Thrombosis also involved the right lateral sinus (not shown). 

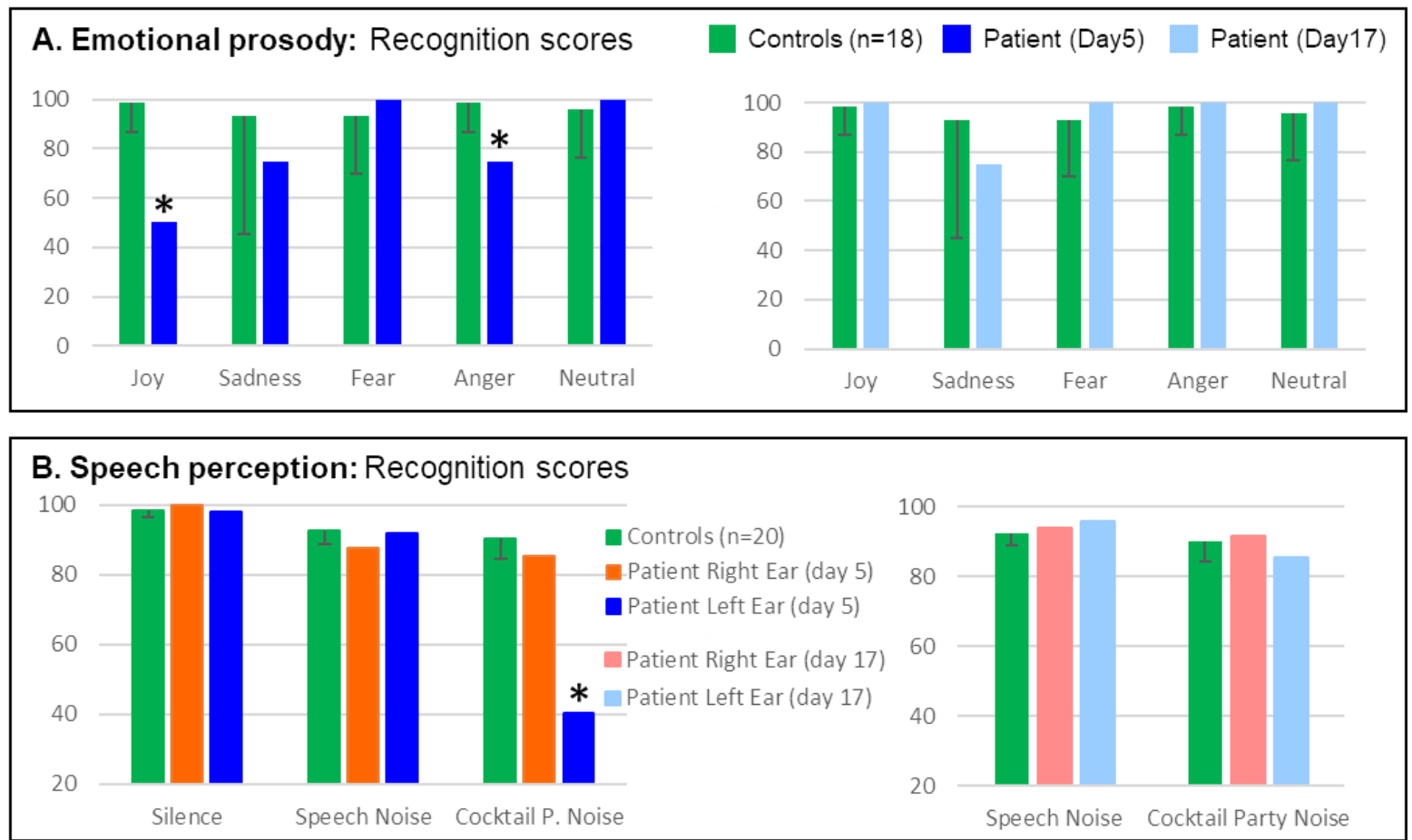

\section{MRI (6 days after admission)}

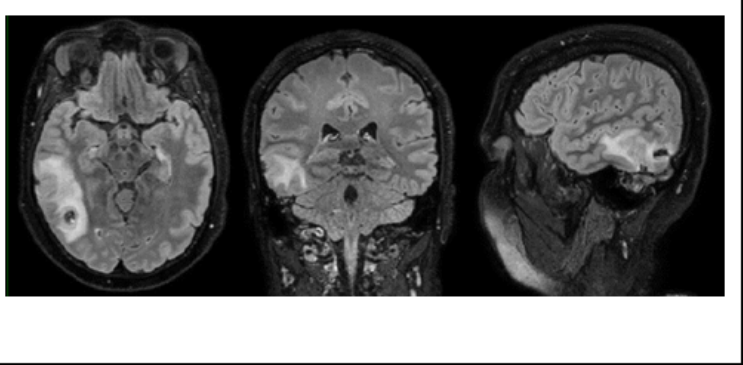

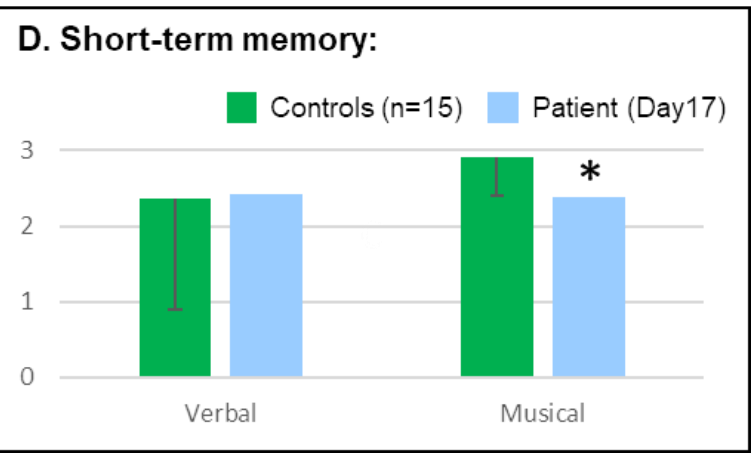

Fig. 2 Results of listening tests performed 5 and 17 days after the patient was admitted to the hospital. a Emotional prosody recognition was assessed with a 5-alternative forced-choice task, with sentences (for the two testing sessions) and vowels (for the second testing session only, data not shown). Data from $n=18$ controls are shown ingreen for comparison. $b$ Speech perception abilities were tested in silence and in different noise types (speech noise and cocktail party, SNR = - $3 d B$ ) for each ear separately (right in orange and left in blue), with a 4-alternative forced-choice task. Data from $n=20$ controls are shown in green for comparison. $c$ Representative slices showing the lesion at 6 days after hospital admission (FLAIR sequence). $d$ Short-term memory was assessed on the second testing session with delayed-matching-to-sample tasks for four-item sequences of verbal (syllables) and musical sounds. Data from $n=15$ controls are shown in green for comparison. For all panels, error bars represent two standard deviations. Patient scores below mean minus two standard deviations of the control groups are marked with an asterisk 


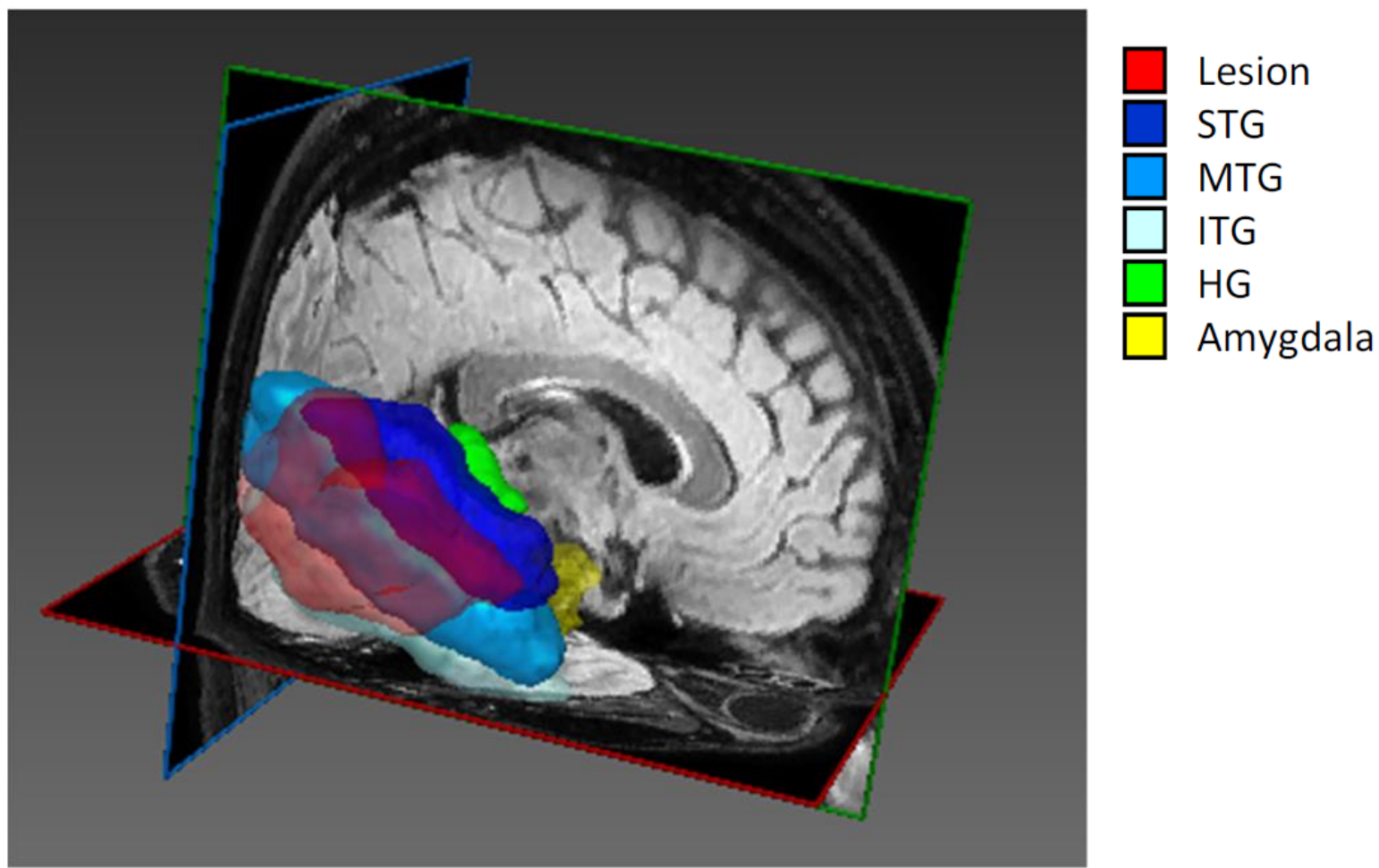

Fig. 3 3D reconstruction of the lesion. The lesion (depicted in red) was reconstructed based on manual drawing on FLAIR images by a trained neurologist using MITK 3M3 (Mint Medical Ins, USA) and then checked against the T1 and T2 images. The lesion included large portions of the superior temporal gyrus (STG, dark blue), middle temporal gyrus (MTG, medium blue), and inferior temporal gyrus (ITG, light blue) in the right hemisphere. However, Heschl's gyrus (HG, in green), as well as mesial temporal structures such as the amygdala (in yellow), were spared. 